\title{
ON NONLOCAL BOUNDARY VALUE PROBLEMS FOR HYPERBOLIC-PARABOLIC EQUATIONS
}

\author{
Allaberen Ashyralyev and Yildirim Ozdemir
}

\begin{abstract}
The nonlocal boundary value problem for hyperbolic-parabolic equations$$
\left\{\begin{array}{c}
\frac{d^{2} u(t)}{d t^{2}}+A u(t)=f(t)(0 \leq t \leq 1), \frac{d u(t)}{d t}+A u(t)=g(t)(-1 \leq t \leq 0), \\
u(-1)=\sum_{i=1}^{N} \alpha_{i} u\left(\mu_{i}\right)+\sum_{i=1}^{L} \beta_{i} u^{\prime}\left(\lambda_{i}\right)+\varphi, \sum_{i=1}^{N}\left|\alpha_{i}\right|, \sum_{i=1}^{L}\left|\beta_{i}\right| \leq 1,0<\mu_{i}, \lambda_{i} \leq 1
\end{array}\right.
$$

for differential equation in a Hilbert space $H$, with the self-adjoint positive definite operator $A$ is considered. The stability estimates for the solution of this problem are established. In applications, the stability estimates for the solutions of the mixed type boundary value problems for hyperbolic-parabolic equations are obtained.
\end{abstract}

\section{INTRODUCTION}

It is known that some problems in fluid mechanics (model of the motion of an ideal fluid filling, exhibiting both viscous and non-viscous phases) and other areas of physics and mathematical biology (taxis-diffusion-reaction model) lead to partial differential equations of the hyperbolic-parabolic type. Methods of solutions of the nonlocal boundary value problems for hyperbolic-parabolic differential equations have been studied extensively by many researchers (see, e.g., [1-12, 22, 23] and the references given therein).

In the present paper we consider the nonlocal boundary value problem

Received July 6, 2005, accepted September 23, 2005.

Communicated by Sen-Yen Shaw.

2000 Mathematics Subject Classification: 65N12, 65M12, 65J10.

Key words and phrases: Hyperbolic-parabolic equation, Nonlocal boundary value problems, Stability. 


$$
\left\{\begin{array}{l}
\frac{d^{2} u(t)}{d t^{2}}+A u(t)=f(t)(0 \leq t \leq 1), \\
\frac{d u(t)}{d t}+A u(t)=g(t)(-1 \leq t \leq 0) \\
u(-1)=\sum_{i=1}^{N} \alpha_{i} u\left(\mu_{i}\right)+\sum_{i=1}^{L} \beta_{i} u^{\prime}\left(\lambda_{i}\right)+\varphi \\
\sum_{i=1}^{N}\left|\alpha_{i}\right|, \sum_{i=1}^{L}\left|\beta_{i}\right| \leq 1,0<\mu_{i}, \lambda_{i} \leq 1
\end{array}\right.
$$

for differential equations of mixed type in a Hilbert space $H$ with self-adjoint positive definite operator $A$.

A function $u(t)$ is called a solution of the problem (1.1) if the following conditions are satisfied:

(i) $u(t)$ is twice continuously differentiable on the interval $(0,1]$ and continuously differentiable on the segment $[-1,1]$. The derivative at the endpoints of the segment are understood as the appropriate unilateral derivatives.

(ii) The element $u(t)$ belongs to $D(A)$ for all $t \in[-1,1]$, and the function $A u(t)$ is continuous on the segment $[-1,1]$.

(iii) $u(t)$ satisfies the equations and nonlocal boundary condition (1.1).

In the paper [18] the following theorem on the stability was proved.

Theorem 1.1. Suppose that $\varphi \in D(A)$ and $f(t)$ be continuously differentiable on $[0,1]$ and $g(t)$ be continuously differentiable on $[-1,0]$ functions and $\alpha_{i}=$ $0, i=2, \cdots, N$ and $\beta_{i}=0, i=1, \cdots, L$. Then there is a unique solution of the problem (1.1) and the stability inequalities

$$
\begin{aligned}
& \max _{-1 \leq t \leq 1}\|u(t)\|_{H} \leq M\left[\|\varphi\|_{H}\right. \\
& \left.\quad+\max _{-1 \leq t \leq 0}\|g(t)\|_{H}+\max _{0 \leq t \leq 1}\left\|A^{-1 / 2} f(t)\right\|_{H}\right], \\
& \max _{-1 \leq t \leq 1}\left\|A^{1 / 2} u(t)\right\|_{H} \leq M\left[\left\|A^{1 / 2} \varphi\right\|_{H}\right. \\
& \left.\quad+\|g(0)\|_{H}+\int_{-1}^{0}\left\|g^{\prime}(t)\right\|_{H} d t+\max _{0 \leq t \leq 1}\|f(t)\|_{H}\right], \\
& \max _{-1 \leq t \leq 0}\left\|\frac{d u(t)}{d t}\right\|_{H}+\max _{0 \leq t \leq 1}\left\|\frac{d^{2} u(t)}{d t^{2}}\right\|_{H} \\
& \quad+\max _{-1 \leq t \leq 1}\|A u(t)\|_{H \leq M} M\left[\|A \varphi\|_{H}+\left\|A^{1 / 2} g(0)\right\|_{H}\right. \\
& \left.\quad+\|f(0)\|_{H}+\max _{-1 \leq t \leq 0}\left\|g^{\prime}(t)\right\|_{H}+\int_{0}^{1}\left\|f^{\prime}(t)\right\|_{H} d t\right]
\end{aligned}
$$

hold, where $M$ does not depend on $f(t), g(t)$ and $\varphi$. 
We have not been able to obtain the same stability estimates for the solutions of the problem (1.1) for $\sum_{i=1}^{L}\left|\beta_{i}\right| \neq 0$. Nevertheless, in the $[23,24]$ the stability estimates for the solution of the problem (1.1) in the cases $\alpha_{i}=0, i=2, \cdots, N$ and $\beta_{i}=0, i=2, \cdots, L$ under a stronger assumption than $f(t)$ be continuously differentiable on $[0,1]$ and $g(t)$ be continuously differentiable on $[-1,0]$ functions are established.

Theorem 1.2. Suppose that $\varphi \in D(A), g(0) \in D\left(A^{\frac{1}{2}}\right), g^{\prime}(0) \in H, f(0) \in$ $D\left(A^{\frac{1}{2}}\right)$ and $f^{\prime}(0) \in H$. Let $f(t)$ be twice continuously differentiable on $[0,1]$ and $g(t)$ be twice continuously differentiable on $[-1,0]$ functions. Then there is a unique solution of the problem (1.1) and the stability inequalities

$$
\begin{aligned}
& \max _{-1 \leq t \leq 1}\|u(t)\|_{H} \leq M\left[\|\varphi\|_{H}+\max _{-1 \leq t \leq 0}\left\|A^{-1 / 2} g^{\prime}(t)\right\|_{H}\right. \\
& \left.+\left\|A^{-1 / 2} g(0)\right\|_{H}+\left\|A^{-1 / 2} f(0)\right\|_{H}+\max _{0 \leq t \leq 1}\left\|A^{-1 / 2} f^{\prime}(t)\right\|_{H}\right], \\
& \max _{-1 \leq t \leq 1}\left\|\frac{d u}{d t}\right\|_{H}+\max _{-1 \leq t \leq 1}\left\|A^{1 / 2} u(t)\right\|_{H} \leq M\left[\left\|A^{1 / 2} \varphi\right\|_{H}\right. \\
& \left.+\|g(0)\|_{H}+\max _{-1 \leq t \leq 0}\left\|g^{\prime}(t)\right\|_{H}+\|f(0)\|_{H}+\max _{0 \leq t \leq 1}\left\|f^{\prime}(t)\right\|_{H}\right], \\
& \max _{-1 \leq t \leq 0}\left\|\frac{d u}{d t}\right\|_{H}+\max _{0 \leq t \leq 1}\left\|\frac{d^{2} u}{d t^{2}}\right\|_{H}+\max _{-1 \leq t \leq 1}\|A u(t)\|_{H} \\
& \leq M\left[\|A \varphi\|_{H}+\left\|A^{1 / 2} g(0)\right\|_{H}+\left\|g^{\prime}(0)\right\|_{H}+\max _{-1 \leq t \leq 0}\left\|g^{\prime \prime}(t)\right\|_{H}\right. \\
& \left.+\left\|A^{1 / 2} f(0)\right\|_{H}+\left\|f^{\prime}(0)\right\|_{H}+\max _{0 \leq t \leq 1}\left\|f^{\prime \prime}(t)\right\|_{H}\right]
\end{aligned}
$$

hold, where $M$ does not depend on $f(t), t \in[0,1], g(t), t \in[-1,0]$ and $\varphi$.

In the present paper the stability estimates for the solution of the problem (1.1) for $\sum_{i=1}^{N}\left|\alpha_{i}\right|, \sum_{i=1}^{L}\left|\beta_{i}\right| \leq 1,0<\mu_{i}, \lambda_{i} \leq 1$ under the assumption that $f(t)$ be twice continuously differentiable on $[0,1]$ and $g(t)$ be twice continuously differentiable on $[-1,0]$ functions are established. In applications, the stability estimates for the solutions of the mixed type boundary value problems for hyperbolic-parabolic equations are obtained.

Finally note that the methods for numerical solutions of the nonlocal boundary value problem (1.1) have been studied extensively (see [14-17, 19-22], and the references therein). 


\section{The Main Theorem}

Let $H$ be a Hilbert space, $A$ be a positive definite self-adjoint operator with $A \geq \delta I$, where $\delta>\delta_{0}>0$. Throughout this paper, $\{c(t), t \geq 0\}$ is a strongly continuous cosine operator-function defined by the formula

$$
c(t)=\frac{e^{i t A^{1 / 2}}+e^{-i t A^{1 / 2}}}{2} .
$$

Then from the definition of the sine operator-function $s(t)$

$$
s(t) u=\int_{0}^{t} c(s) u d s
$$

it follows that

$$
s(t)=A^{-1 / 2} \frac{e^{i t A^{1 / 2}}-e^{-i t A^{1 / 2}}}{2 i} .
$$

For the theory of cosine operator-function we refer to [25] and [26].

Now, let us give some lemmas that will be needed below.

Lemma 2.1. The estimates hold:

$$
\begin{gathered}
\|c(t)\|_{H \rightarrow H} \leq 1,\left\|A^{1 / 2} s(t)\right\|_{H \rightarrow H} \leq 1, \\
\left\|A^{\gamma} e^{-t A}\right\|_{H \rightarrow H} \leq t^{-\gamma} e^{-\delta t}, t>0,0 \leq \gamma \leq 1, \delta>0 .
\end{gathered}
$$

Lemma 2.2. The operator

$$
I-\sum_{i=1}^{N} \alpha_{i}\left[c\left(\mu_{i}\right)-A s\left(\mu_{i}\right)\right] e^{-A}+\sum_{i=1}^{L} \beta_{i}\left[s\left(\lambda_{i}\right)+c\left(\lambda_{i}\right)\right] A e^{-A}
$$

has an inverse

$$
T=\left(I-\sum_{i=1}^{N} \alpha_{i}\left[c\left(\mu_{i}\right)-A s\left(\mu_{i}\right)\right] e^{-A}+\sum_{i=1}^{L} \beta_{i}\left[s\left(\lambda_{i}\right)+c\left(\lambda_{i}\right)\right] A e^{-A}\right)^{-1}
$$

and the following estimate

$$
\|T\|_{H \rightarrow H} \leq M
$$


holds, where $M$ does not depend on $\alpha_{i}, \beta_{i}, \mu_{i}$ and $\lambda_{i}$.

Proof. The proof of the estimate (2.3) is based on the estimate

$$
\left\|-\sum_{i=1}^{N} \alpha_{i}\left[c\left(\mu_{i}\right)-A s\left(\mu_{i}\right)\right] e^{-A}+\sum_{i=1}^{L} \beta_{i}\left[s\left(\lambda_{i}\right)+c\left(\lambda_{i}\right)\right] A e^{-A}\right\|_{H \rightarrow H}<1 .
$$

Using the definitions of $c(\mu)$ and $s(\mu)$ and positivity and self-adjointness property of $A$, we obtain

$$
\begin{aligned}
& \left\|-\sum_{i=1}^{N} \alpha_{i}\left[c\left(\mu_{i}\right)-A s\left(\mu_{i}\right)\right] e^{-A}+\sum_{i=1}^{L} \beta_{i}\left[s\left(\lambda_{i}\right)+c\left(\lambda_{i}\right)\right] A e^{-A}\right\|_{H \rightarrow H} \\
\leq & \sup _{\delta \leq \rho<\infty} \mid-\sum_{i=1}^{N} \alpha_{i}\left[\cos \left(\sqrt{\rho} \mu_{i}\right)-\sqrt{\rho} \sin \left(\sqrt{\rho} \mu_{i}\right)\right] \\
& +\sum_{i=1}^{L} \beta_{i}\left[\sqrt{\rho} \sin \left(\sqrt{\rho} \lambda_{i}\right)+\rho \cos \left(\sqrt{\rho} \lambda_{i}\right)\right] \mid e^{-\rho} .
\end{aligned}
$$

Since

$$
\begin{aligned}
& \cos \left(\sqrt{\rho} \mu_{i}\right)-\sqrt{\rho} \sin \left(\sqrt{\rho} \mu_{i}\right)=\sqrt{\rho+1} \cos \left(\sqrt{\rho} \mu_{i}-\mu_{i 0}\right), \\
& \sqrt{\rho} \sin \left(\sqrt{\rho} \lambda_{i}\right)+\rho \cos \left(\sqrt{\rho} \lambda_{i}\right)=\sqrt{\rho} \sqrt{\rho+1} \cos \left(\sqrt{\rho} \lambda_{i}-\lambda_{i 0}\right),
\end{aligned}
$$

we have that

$$
\begin{aligned}
& \left\|-\sum_{i=1}^{N} \alpha_{i}\left[c\left(\mu_{i}\right)-A s\left(\mu_{i}\right)\right] e^{-A}+\sum_{i=1}^{L} \beta_{i}\left[s\left(\lambda_{i}\right)+c\left(\lambda_{i}\right)\right] A e^{-A}\right\|_{H \rightarrow H} \\
\leq & \sup _{\delta \leq \rho<\infty}\left[\sum_{i=1}^{N}\left|\alpha_{i}\right|\left|\cos \left(\sqrt{\rho} \mu_{i}\right)-\sqrt{\rho} \sin \left(\sqrt{\rho} \mu_{i}\right)\right|\right. \\
& \left.+\sum_{i=1}^{L}\left|\beta_{i}\right|\left|\sqrt{\rho} \sin \left(\sqrt{\rho} \lambda_{i}\right)+\rho \cos \left(\sqrt{\rho} \lambda_{i}\right)\right|\right] e^{-\rho} . \\
\leq & \sup _{\delta \leq \rho<\infty}\left[\sum_{i=1}^{N}\left|\alpha_{i}\right| \sqrt{\rho+1}+\sum_{i=1}^{L}\left|\beta_{i}\right| \sqrt{\rho} \sqrt{\rho+1}\right] e^{-\rho} \\
\leq & \sup _{0<\delta_{0}<\delta \leq \rho<\infty} \sqrt{\rho+1}(1+\sqrt{\rho}) e^{-\rho} .
\end{aligned}
$$

Since $\sup _{0<\delta_{0}<\delta \leq \rho<\infty} \sqrt{\rho+1}(1+\sqrt{\rho}) e^{-\rho}<1$, we have the estimate (2.4). Lemma 2.2 is proved.

Now, we will obtain the formula for solution of the problem (1.1). It is known that for smooth data of the initial value problems

$$
\left\{\begin{array}{c}
u^{\prime \prime}(t)+A u(t)=f(t), \quad(0 \leq t \leq 1) \\
u(0)=u_{0}, u^{\prime}(0)=u_{0}^{\prime}
\end{array}\right.
$$




$$
\left\{\begin{array}{c}
u^{\prime}(t)+A u(t)=g(t), \quad(-1 \leq t \leq 0), \\
u(-1)=u_{-1},
\end{array}\right.
$$

there are unique solutions of the problems (2.5), (2.6) and the following formulas hold:

$$
u(t)=c(t) u(0)+s(t) u^{\prime}(0)+\int_{0}^{t} s(t-y) f(y) d y, \quad 0 \leq t \leq 1,
$$

and

$$
u(t)=e^{-(t+1) A} u_{-1}+\int_{-1}^{t} e^{-(t-y) A} g(y) d y, \quad-1 \leq t \leq 0 .
$$

Using formulas (2.7), (2.8) and equation (1.1) we can write

$$
\begin{aligned}
u(t) & =[c(t)-A s(t)]\left\{e^{-A} u_{-1}+\int_{-1}^{0} e^{y A} g(y) d y\right\} \\
& +s(t) g(0)+\int_{0}^{t} s(t-y) f(y) d y .
\end{aligned}
$$

Now, using the condition

$$
u(-1)=\sum_{i=1}^{N} \alpha_{i} u\left(\mu_{i}\right)+\sum_{i=1}^{L} \beta_{i} u^{\prime}\left(\lambda_{i}\right)+\varphi,
$$

we obtain the operator equation

$$
\begin{aligned}
& \left\{I-\sum_{i=1}^{N} \alpha_{i}\left[c\left(\mu_{i}\right)-A s\left(\mu_{i}\right)\right] e^{-A}+\sum_{i=1}^{L} \beta_{i}\left[s\left(\lambda_{i}\right)+c\left(\lambda_{i}\right)\right] A e^{-A}\right\} u_{-1} \\
= & \sum_{i=1}^{N} \alpha_{i}\left\{\left[c\left(\mu_{i}\right)-A s\left(\mu_{i}\right)\right] \int_{-1}^{0} e^{y A} g(y) d y\right. \\
(2.10)+ & \left.s\left(\mu_{i}\right) g(0)+\int_{0}^{\mu_{i}} s\left(\mu_{i}-y\right) f(y) d y\right\} \\
+ & \sum_{i=1}^{L} \beta_{i}\left\{\left[-A s\left(\lambda_{i}\right)-A c\left(\lambda_{i}\right)\right] \int_{-1}^{0} e^{y A} g(y) d y\right. \\
+ & \left.c\left(\lambda_{i}\right) g(0)+\int_{0}^{\lambda_{i}} c\left(\lambda_{i}-y\right) f(y) d y\right\}+\varphi .
\end{aligned}
$$

Since the operator

$$
I-\sum_{i=1}^{N} \alpha_{i}\left[c\left(\mu_{i}\right)-A s\left(\mu_{i}\right)\right] e^{-A}+\sum_{i=1}^{L} \beta_{i}\left[s\left(\lambda_{i}\right)+c\left(\lambda_{i}\right)\right] A e^{-A}
$$


has an inverse

$$
T=\left(I-\sum_{i=1}^{N} \alpha_{i}\left[c\left(\mu_{i}\right)-A s\left(\mu_{i}\right)\right] e^{-A}+\sum_{i=1}^{L} \beta_{i}\left[s\left(\lambda_{i}\right)+c\left(\lambda_{i}\right)\right] A e^{-A}\right)^{-1},
$$

for the solution of the operator equation (2.10) we have the formula

$$
\begin{aligned}
& u_{-1}=T\left[\sum _ { i = 1 } ^ { N } \alpha _ { i } \left\{\left[c\left(\mu_{i}\right)-A s\left(\mu_{i}\right)\right] \int_{-1}^{0} e^{y A} g(y) d y\right.\right. \\
& \left.+s\left(\mu_{i}\right) g(0)+\int_{0}^{\mu_{i}} s\left(\mu_{i}-y\right) f(y) d y\right\} \\
& +\sum_{i=1}^{L} \beta_{i}\left\{\left[-A s\left(\lambda_{i}\right)-A c\left(\lambda_{i}\right)\right] \int_{-1}^{0} e^{y A} g(y) d y\right. \\
& \left.\left.+c\left(\lambda_{i}\right) g(0)+\int_{0}^{\lambda_{i}} c\left(\lambda_{i}-y\right) f(y) d y\right\}+\varphi\right] .
\end{aligned}
$$

Hence, for the solution of the nonlocal boundary value problem (1.1) we have the formulas (2.8), (2.9) and (2.11).

Theorem 2.1. Suppose that $\varphi \in D(A), g(0) \in D\left(A^{\frac{1}{2}}\right), g^{\prime}(0) \in H, f(0) \in$ $D\left(A^{\frac{1}{2}}\right)$ and $f^{\prime}(0) \in H$. Let $f(t)$ be twice continuously differentiable on $[0,1]$ and $g(t)$ be twice continuously differentiable on $[-1,0]$ functions. Then there is a unique solution of the problem (1.1) and the following stability inequalities

$$
\begin{aligned}
& \max _{-1 \leq t \leq 1}\|u(t)\|_{H} \leq M\left[\|\varphi\|_{H}+\max _{-1 \leq t \leq 0}\left\|A^{-1 / 2} g^{\prime}(t)\right\|_{H}\right. \\
& \left.+\left\|A^{-1 / 2} g(0)\right\|_{H}+\left\|A^{-1 / 2} f(0)\right\|_{H}+\max _{0 \leq t \leq 1}\left\|A^{-1 / 2} f^{\prime}(t)\right\|_{H}\right], \\
& \max _{-1 \leq t \leq 1}\left\|\frac{d u}{d t}\right\|_{H}+\max _{-1 \leq t \leq 1}\left\|A^{1 / 2} u(t)\right\|_{H} \leq M\left[\left\|A^{1 / 2} \varphi\right\|_{H}\right. \\
& \left.+\|g(0)\|_{H}+\max _{-1 \leq t \leq 0}\left\|g^{\prime}(t)\right\|_{H}+\|f(0)\|_{H}+\max _{0 \leq t \leq 1}\left\|f^{\prime}(t)\right\|_{H}\right], \\
& \max _{-1 \leq t \leq 0}\left\|\frac{d u}{d t}\right\|_{H}+\max _{0 \leq t \leq 1}\left\|\frac{d^{2} u}{d t^{2}}\right\|_{H}+\max _{-1 \leq t \leq 1}\|A u(t)\|_{H} \\
& \leq M\left[\|A \varphi\|_{H}+\left\|A^{1 / 2} g(0)\right\|_{H}+\left\|g^{\prime}(0)\right\|_{H}+\max _{-1 \leq t \leq 0}\left\|g^{\prime \prime}(t)\right\|_{H}\right. \\
& \left.+\left\|A^{1 / 2} f(0)\right\|_{H}+\left\|f^{\prime}(0)\right\|_{H}+\max _{0 \leq t \leq 1}\left\|f^{\prime \prime}(t)\right\|_{H}\right]
\end{aligned}
$$

hold, where $M$ does not depend on $f(t), t \in[0,1], g(t), t \in[-1,0]$ and $\varphi$.

Proof. First, we obtain estimate (2.12). Using formula (2.11) and an integration by parts, we obtain 


$$
\begin{aligned}
& u_{-1}=T\left[\sum _ { i = 1 } ^ { N } \alpha _ { i } \left\{c ( \mu _ { i } ) \left[A ^ { - 1 } \left(g(0)-e^{-A} g(-1)\right.\right.\right.\right. \\
& \left.\left.-\int_{-1}^{0} e^{y A} g^{\prime}(y) d y\right)\right]+A s\left(\mu_{i}\right)\left(e^{-A} g(-1)+\int_{-1}^{0} e^{y A} g^{\prime}(y) d y\right) \\
& \left.+A^{-1}\left[f\left(\mu_{i}\right)-c\left(\mu_{i}\right) f(0)-\int_{0}^{\mu_{i}} c\left(\mu_{i}-y\right) f^{\prime}(y) d y\right]\right\} \\
& +\sum_{i=1}^{L} \beta_{i}\left\{-A s\left(\lambda_{i}\right)\left(g(0)-e^{-A} g(-1)-\int_{-1}^{0} e^{y A} g^{\prime}(y) d y\right)\right. \\
& +A c\left(\lambda_{i}\right)\left(e^{-A} g(-1)+\int_{-1}^{0} e^{y A} g^{\prime}(y) d y\right) \\
& \left.\left.+A s\left(\lambda_{i}\right) f(0)+\int_{0}^{\lambda_{i}} s\left(\lambda_{i}-y\right) f^{\prime}(y) d y\right\}+\varphi\right] .
\end{aligned}
$$

Using estimates (2.3), (2.1) and (2.2), we obtain

$$
\begin{aligned}
& \left\|u_{-1}\right\|_{H} \leq M\left[\|\varphi\|_{H}+\max _{-1 \leq t \leq 0}\left\|A^{-1 / 2} g^{\prime}(t)\right\|_{H}\right. \\
& \left.\quad+\left\|A^{-1 / 2} g(0)\right\|_{H}+\left\|A^{-1 / 2} f(0)\right\|_{H}+\max _{0 \leq t \leq 1}\left\|A^{-1 / 2} f^{\prime}(t)\right\|_{H}\right] .
\end{aligned}
$$

Using formulas (2.8), (2.9) and an integration by parts, we obtain

$$
u(t)=e^{-(t+1) A} u_{-1}+A^{-1}\left(g(t)-e^{-A} g(-1)\right.
$$

$$
\left.-\int_{-1}^{t} e^{y A} g^{\prime}(y) d y, \quad-1 \leq t \leq 0,\right)
$$

$$
\begin{aligned}
& u(t)=[c(t)-A s(t)]\left\{e^{-A} u_{-1}\right. \\
& \left.+A^{-1}\left(g(0)-e^{-A} g(-1)-\int_{-1}^{0} e^{y A} g^{\prime}(y) d y\right)\right\}+s(t) g(0) \\
& +A^{-1}\left[f(t)-c(t) f(0)-\int_{0}^{t} c(t-y) f^{\prime}(y) d y, 0 \leq t \leq 1 .\right]
\end{aligned}
$$

Using estimates (2.1) and (2.2), we obtain

$$
\begin{gathered}
\|u(t)\|_{H} \leq M\left[\left\|u_{-1}\right\|_{H}+\max _{-1 \leq t \leq 0}\left\|A^{-1 / 2} g^{\prime}(t)\right\|_{H}+\left\|A^{-1 / 2} g(0)\right\|_{H}\right],-1 \leq t \leq 0, \\
\|u(t)\|_{H} \leq M\left[\max _{0 \leq t \leq 1}\left\|A^{-1 / 2} f^{\prime}(t)\right\|_{H}+\left\|A^{-1 / 2} f(0)\right\|_{H}\right. \\
\left.+\left\|u_{-1}\right\|_{H}+\max _{-1 \leq t \leq 0}\left\|A^{-1 / 2} g^{\prime}(t)\right\|_{H}+\left\|A^{-1 / 2} g(0)\right\|_{H}\right], \quad 0 \leq t \leq 1 .
\end{gathered}
$$


Then from (2.16) and the last two estimates, it follows (2.12).

Second, we obtain estimate (2.13). Applying $A^{1 / 2}$ to the formula (2.15) and using estimates (2.3), (2.1) and (2.2), we obtain

$$
\begin{gathered}
\left\|A^{1 / 2} u_{-1}\right\|_{H} \leq M\left[\left\|A^{1 / 2} \varphi\right\|_{H}+\max _{-1 \leq t \leq 0}\left\|g^{\prime}(t)\right\|_{H}\right. \\
\left.+\|g(0)\|_{H}+\|f(0)\|_{H}+\max _{0 \leq t \leq 1}\left\|f^{\prime}(t)\right\|_{H}\right]
\end{gathered}
$$

Applying $A^{1 / 2}$ to the formulas (2.17), (2.18) and using estimates (2.1) and (2.2), we obtain

$$
\begin{aligned}
& \left\|A^{1 / 2} u(t)\right\|_{H} \leq M\left[\left\|A^{1 / 2} u_{-1}\right\|_{H}+\max _{-1 \leq t \leq 0}\left\|g^{\prime}(t)\right\|_{H}+\|g(0)\|_{H}\right], \quad-1 \leq t \leq 0, \\
& \left\|A^{1 / 2} u(t)\right\|_{H} \leq M\left[\max _{0 \leq t \leq 1}\left\|f^{\prime}(t)\right\|_{H}+\|f(0)\|_{H}\right. \\
& \left.\quad+\left\|A^{1 / 2} u_{-1}\right\|_{H}+\max _{-1 \leq t \leq 0}\left\|g^{\prime}(t)\right\|_{H}+\|g(0)\|_{H}\right], \quad 0 \leq t \leq 1 .
\end{aligned}
$$

Then from (2.19) and the last two estimates, it follows (2.13).

Third, we obtain estimate (2.14). Using formula (2.15) and an integration by parts, we obtain

$$
\begin{aligned}
u_{-1} & =T\left[\sum _ { i = 1 } ^ { N } \alpha _ { i } \left\{c ( \mu _ { i } ) \left[A ^ { - 1 } \left(g(0)-e^{-A} g(-1)\right.\right.\right.\right. \\
& \left.-A^{-1}\left[g^{\prime}(0)-e^{-A} g^{\prime}(-1)-\int_{-1}^{0} e^{y A} g^{\prime \prime}(y) d y\right]\right) \\
& +A s\left(\mu_{i}\right)\left(e^{-A} g(-1)+A^{-1}\left[g^{\prime}(0)-e^{-A} g^{\prime}(-1)-\int_{-1}^{0} e^{y A} g^{\prime \prime}(y) d y\right]\right) \\
& \left.+A^{-1}\left[f\left(\mu_{i}\right)-c\left(\mu_{i}\right) f(0)-\left[s\left(\mu_{i}\right) f^{\prime}(0)-\int_{0}^{\mu} s\left(\mu_{i}-y\right) f^{\prime \prime}(y) d y\right]\right]\right\} \\
& +\sum_{i=1}^{L} \beta_{i}\left\{-A s\left(\lambda_{i}\right)\left(g(0)-e^{-A} g(-1)-A^{-1}\left[g^{\prime}(0)-e^{-A} g^{\prime}(-1)-\int_{-1}^{0} e^{y A} g^{\prime \prime}(y) d y\right]\right)\right. \\
& +A c\left(\lambda_{i}\right)\left(e^{-A} g(-1)+A^{-1}\left[g^{\prime}(0)-e^{-A} g^{\prime}(-1)-\int_{-1}^{0} e^{y A} g^{\prime \prime}(y) d y\right]\right) \\
& \left.\left.+A s\left(\lambda_{i}\right) f(0)-A^{-1}\left[f^{\prime}\left(\lambda_{i}\right)-c\left(\lambda_{i}\right) f^{\prime}(0)-\int_{0}^{\lambda} c\left(\lambda_{i}-y\right) f^{\prime \prime}(y) d y\right]\right\}+\varphi\right] .
\end{aligned}
$$


Using estimates (2.3), (2.1) and (2.2), we obtain

$$
\begin{aligned}
& \left\|A u_{-1}\right\|_{H} \leq M\left[\|A \varphi\|_{H}+\max _{-1 \leq t \leq 0}\left\|g^{\prime \prime}(t)\right\|_{H}+\left\|g^{\prime}(0)\right\|_{H}\right. \\
& \left.\quad+\left\|A^{1 / 2} g(0)\right\|_{H}+\left\|A^{1 / 2} f(0)\right\|_{H}+\left\|f^{\prime}(0)\right\|_{H}+\max _{0 \leq t \leq 1}\left\|f^{\prime \prime}(t)\right\|_{H}\right] .
\end{aligned}
$$

Using formulas (2.17), (2.18) and an integration by parts, we obtain

$$
\begin{aligned}
u(t) & =e^{-(t+1) A} u_{-1}+A^{-1}\left(g(t)-e^{-A} g(-1)\right. \\
& \left.-A^{-1}\left[g^{\prime}(t)-e^{-A} g^{\prime}(-1)-\int_{-1}^{t} e^{y A} g^{\prime \prime}(y) d y\right]\right),-1 \leq t \leq 0, \\
u(t) & =[c(t)-A s(t)]\left\{e^{-A} u_{-1}+A^{-1}\left(g(0)-e^{-A} g(-1)\right.\right. \\
& \left.\left.-A^{-1}\left[g^{\prime}(0)-e^{-A} g^{\prime}(-1)-\int_{-1}^{0} e^{y A} g^{\prime \prime}(y) d y\right]\right)\right\} \\
& +s(t) g(0)+A^{-1} f(t)-c(t) f(0) \\
& -\left[s(t) f^{\prime}(0)+\int_{0}^{t} s(t-y) f^{\prime \prime}(y) d y\right], 0 \leq t \leq 1 .
\end{aligned}
$$

Applying $A$ to the last two formulas and using estimates (2.1) and (2.2), we obtain

$$
\begin{aligned}
& \|A u(t)\|_{H} \leq M\left[\left\|A u_{-1}\right\|_{H}+\max _{-1 \leq t \leq 0}\left\|g^{\prime \prime}(t)\right\|_{H}+\left\|A^{1 / 2} g(0)\right\|_{H}+\left\|g^{\prime}(0)\right\|_{H}\right], \\
& -1 \leq t \leq 0 \\
& \|A u(t)\|_{H} \leq M\left[\max _{0 \leq t \leq 1}\left\|f^{\prime \prime}(t)\right\|_{H}+\left\|A^{1 / 2} f(0)\right\|_{H}+\left\|f^{\prime}(0)\right\|_{H}\right. \\
& \left.\quad+\left\|A u_{-1}\right\|_{H}+\max _{-1 \leq t \leq 0}\left\|g^{\prime \prime}(t)\right\|_{H}+\left\|A^{1 / 2} g(0)\right\|_{H}+\left\|g^{\prime}(0)\right\|_{H}\right], \quad 0 \leq t \leq 1 .
\end{aligned}
$$

Then from (2.20) and the last two estimates, it follows (2.14). Theorem 2.1 is proved.

Remark 1. Applying this approach we have not been able to establish the same stability estimates for the solution of the problem (1.1) when an operator $T$ is unbounded or not exist.

\section{Applications}

First, we consider the mixed problem for hyperbolic- parabolic equation 


$$
\left\{\begin{array}{l}
v_{t t}-\left(a(x) v_{x}\right)_{x}+\delta v=f(t, x), 0<t<1,0<x<1 \\
v_{t}-\left(a(x) v_{x}\right)_{x}+\delta v=g(t, x),-1<t<0,0<x<1 \\
v(-1, x)=\sum_{i=1}^{N} \alpha_{i} v\left(\mu_{i}, x\right)+\sum_{i=1}^{L} \beta_{i} v_{t}\left(\lambda_{i}, x\right)+\varphi(x), 0 \leq x \leq 1 \\
\sum_{i=1}^{N}\left|\alpha_{i}\right|, \sum_{i=1}^{L}\left|\beta_{i}\right| \leq 1,0<\mu_{i}, \lambda_{i} \leq 1 \\
v(t, 0)=v(t, 1), v_{x}(t, 0)=v_{x}(t, 1),-1 \leq t \leq 1 \\
v(0+, x)=v(0-, x), v_{t}(0+, x)=v_{t}(0-, x), 0 \leq x \leq 1
\end{array}\right.
$$

Problem (3.1) has a unique smooth solution $v(t, x)$ for the smooth $a(x) \geq a>$ $0(x \in(0,1)), \varphi(x) \quad(x \in[0,1])$ and $f(t, x)(t \in[0,1], x \in[0,1]), g(t, x)(t \in$ $[-1,0], x \in[0,1])$ functions and $\delta=$ const $>0$. This allows us to reduce the mixed problem (3.1) to the nonlocal boundary value problem (1.1) in Hilbert space $H$ with a self-adjoint positive definite operator $A$ defined by (3.1). Let us give a number of corollaries of the abstract Theorem 2.1.

Theorem 3.1. The solutions of the nonlocal boundary value problem (3.1) satisfy the stability estimates

$$
\begin{aligned}
& \max _{-1 \leq t \leq 1}\|v(t)\|_{L_{2}[0,1]} \leq M\left[\|f(0)\|_{L_{2}[0,1]}+\max _{0 \leq t \leq 1}\left\|f_{t}(t)\right\|_{L_{2}[0,1]}\right. \\
& \left.\quad+\|g(0)\|_{L_{2}[0,1]}+\max _{-1 \leq t \leq 0}\left\|g_{t}(t)\right\|_{L_{2}[0,1]}+\|\varphi\|_{L_{2}[0,1]}\right] \\
& \max _{-1 \leq t \leq 1}\|v(t)\|_{W_{2}^{1}[0,1]} \leq M\left[\|f(0)\|_{L_{2}[0,1]}+\max _{0 \leq t \leq 1}\left\|f_{t}(t)\right\|_{L_{2}[0,1]}\right. \\
& \left.\quad+\|g(0)\|_{L_{2}[0,1]}+\max _{-1 \leq t \leq 0}\left\|g_{t}(t)\right\|_{L_{2}[0,1]}+\|\varphi\|_{W_{2}^{1}[0,1]}\right] \\
& \max _{-1 \leq t \leq 1}\|v(t)\|_{W_{2}^{2}[0,1]}+\max _{-1 \leq t \leq 0}\left\|v_{t}(t)\right\|_{L_{2}[0,1]}+\max _{0 \leq t \leq 1}\left\|v_{t t}(t)\right\|_{L_{2}[0,1]} \\
& \quad \leq M\left[\|\varphi\|_{W_{2}^{2}[0,1]}+\|f(0)\|_{W_{2}^{1}[0,1]}+\left\|f_{t}(0)\right\|_{L_{2}[0,1]}+\max _{0 \leq t \leq 1}\left\|f_{t t}(t)\right\|_{L_{2}[0,1]}\right. \\
& \left.\quad+\|g(0)\|_{W_{2}^{1}[0,1]}+\left\|g_{t}(0)\right\|_{L_{2}[0,1]}+\max _{-1 \leq t \leq 0}\left\|g_{t t}(t)\right\|_{L_{2}[0,1]}\right]
\end{aligned}
$$

where $M$ does not depend on $f(t, x)(t \in[0,1], x \in[0,1]), g(t, x)(t \in[-1,0], x \in$ $[0,1])$ and $\varphi(x)(x \in[0,1])$.

The proof of this theorem is based on the abstract Theorem 2.1 and the symmetry properties of the space operator generated by problem (3.1). 
Second, let $\Omega$ be the unit open cube in the n-dimensional Euclidean space $R^{n}\left(0<x_{k}<1,1 \leq k \leq n\right)$ with boundary $S, \bar{\Omega}=\Omega \cup S$. In $[0,1] \times \Omega$ we consider the mixed boundary value problem for the multidimensional hyperbolicparabolic equation

$$
\left\{\begin{array}{l}
v_{t t}-\sum_{r=1}^{n}\left(a_{r}(x) v_{x_{r}}\right)_{x_{r}}=f(t, x), 0 \leq t \leq 1 \\
x=\left(x_{1}, \ldots, x_{n}\right) \in \Omega \\
v_{t}-\sum_{r=1}^{n}\left(a_{r}(x) v_{x_{r}}\right)_{x_{r}}=g(t, x),-1 \leq t \leq 0 \\
x=\left(x_{1}, \ldots, x_{n}\right) \in \Omega \\
v(-1, x)=\sum_{i=1}^{N} \alpha_{i} v\left(\mu_{i}, x\right)+\sum_{i=1}^{L} \beta_{i} v_{t}\left(\lambda_{i}, x\right)+\varphi(x), x \in \bar{\Omega} \\
\sum_{i=1}^{N}\left|\alpha_{i}\right|, \sum_{i=1}^{L}\left|\beta_{i}\right| \leq 1,0<\mu_{i}, \lambda_{i} \leq 1 \\
u(t, x)=0, x \in S,-1 \leq t \leq 1 \\
v(0+, x)=v(0-, x), v_{t}(0+, x)=v_{t}(0-, x), x \in \bar{\Omega} .
\end{array}\right.
$$

We introduce the Hilbert spaces $L_{2}(\bar{\Omega})$ of the all integrable functions defined on $\bar{\Omega}$, equipped with the norm

$$
\|f\|_{L_{2}(\bar{\Omega})}=\left\{\int \cdots \int_{x \in \bar{\Omega}}|f(x)|^{2} d x_{1} \cdots d x_{n}\right\}^{1 / 2} .
$$

Problem (3.2) has a unique smooth solution $v(t, x)$ for the smooth $a_{r}(x) \geq a>0$, $\varphi(x)(x \in \bar{\Omega})$ and $f(t, x)(t \in(0,1), x \in \Omega), g(t, x)(t \in(-1,0), x \in \Omega)$ functions. This allows us to reduce the mixed problem (3.2) to the nonlocal boundary value problem (1.1) in Hilbert space $H$ with a self- adjoint positive definite operator $A$ defined by (3.2). Let us give a number of corollaries of the abstract Theorem 2.1 .

Theorem 3.2. The solutions of the nonlocal boundary value problem (3.2) satisfy the stability estimates

$$
\begin{aligned}
& \max _{-1 \leq t \leq 1}\|v(t)\|_{L_{2}(\bar{\Omega})} \leq M\left[\|f(0)\|_{L_{2}(\bar{\Omega})}+\max _{0 \leq t \leq 1}\left\|f_{t}(t)\right\|_{L_{2}(\bar{\Omega})}\right. \\
& \left.\quad+\|g(0)\|_{L_{2}(\bar{\Omega})}+\max _{-1 \leq t \leq 0}\left\|g_{t}(t)\right\|_{L_{2}(\bar{\Omega})}+\|\varphi\|_{L_{2}(\bar{\Omega})}\right], \\
& \max _{-1 \leq t \leq 1}\|v(t)\|_{W_{2}^{1}(\bar{\Omega})} \leq M\left[\|f(0)\|_{L_{2}(\bar{\Omega})}+\max _{0 \leq t \leq 1}\left\|f_{t}(t)\right\|_{L_{2}(\bar{\Omega})}\right.
\end{aligned}
$$




$$
\begin{aligned}
& \left.\quad+\|g(0)\|_{L_{2}(\bar{\Omega})}+\max _{-1 \leq t \leq 0}\left\|g_{t}(t)\right\|_{L_{2}(\bar{\Omega})}+\|\varphi\|_{W_{2}^{1}(\bar{\Omega})}\right] \\
& \max _{-1 \leq t \leq 1}\|v(t)\|_{W_{2}^{2}(\bar{\Omega})}+\max _{-1 \leq t \leq 0}\left\|v_{t}(t)\right\|_{L_{2}(\bar{\Omega})}+\max _{0 \leq t \leq 1}\left\|v_{t t}(t)\right\|_{L_{2}(\bar{\Omega})} \\
& \leq M \mid\left[\left.\|\varphi\|\right|_{W_{2}^{2}(\bar{\Omega})}+\|f(0)\|_{W_{2}^{1}(\bar{\Omega})}+\left\|f_{t}(0)\right\|_{L_{2}(\bar{\Omega})}+\max _{0 \leq t \leq 1}\left\|f_{t t}(t)\right\|_{L_{2}(\bar{\Omega})}\right. \\
& \left.\quad+\|g(0)\|_{W_{2}^{1}(\bar{\Omega})}+\left\|g_{t}(0)\right\|_{L_{2}(\bar{\Omega})}+\max _{-1 \leq t \leq 0}\left\|g_{t t}(t)\right\|_{L_{2}(\bar{\Omega})}\right]
\end{aligned}
$$

where $M$ does not depend on $f(t, x)(t \in[0,1], x \in \bar{\Omega}), g(t, x)(t \in[-1,0], x \in \bar{\Omega}])$ and $\varphi(x)(x \in \bar{\Omega})$.

The proof of this theorem is based on the abstract Theorem 2.1 and the symmetry properties of the space operator generated by problem (3.2).

\section{ACKNOWLEDGMENT}

The authors would like to thank Prof. P. E. Sobolevskii for his helpful suggestions to the improvement of this paper.

\section{REFERENCES}

1. V. I. Korzyuk and S. V. Lemeshevsky, Problems on conjugation of polytypic equations, Mathematical Modelling and Analysis, 6(1) (2001), 106-116.

2. G. Vallet, Weak entropic solution to a scalar hyperbolic-parabolic conservation law, Rev. R. Acad. Cien. Serie A. Mat., 97(1) (2003), 147-152.

3. S. N. Glazatov, Nonlocal boundary value problems for linear and nonlinear equations of variable type, Sobolev Institue of Mathematics SB RAS, Preprint 46 (1998), p. 26.

4. G. D. Karatoprakliev, On a nonlocal boundary value problem for hyperbolic-parabolic equations, Differensial'nye uravneniya (in Russian), 25(8) (1989), 1355-1359.

5. M. G. Karatopraklieva, On a nonlocal boundary value problem for an equation of mixed type, Differensial'nye uravneniya (in Russian), 27(1) (1991), 68-79.

6. G. Q. Chen, and E. Dibenedetto, Stability of entropy solutions to the Cauchy problem for a class of nonlinear hyperbolic-parabolic equations.

7. A. Gerish, M. Kotschote and R. Zacher, Well-posed of a quasilinear hyperbolicparabolic system arising in mathematical biology, Report on Analysis and Numerical Mathematics, Martin-Luther-Universitat Halle-Wittenberg, Germany, no. 04-24, August 31, 2004.

8. M. S. Salahatdinov, Equations of Mixed-Composite Type, Tashkent: FAN (in Russian), 1974, p. 156. 
9. T. D. Djuraev, Boundary Value Problems for Equations of Mixed and Mixed-Composite Types, Tashkent: FAN (in Russian), 1979, p. 238.

10. D. Bazarov and H. Soltanov, Some Local and Nonlocal Boundary Value Problems for Equations of Mixed and Mixed-Composite Types, Ashgabat: Ylym (in Russian), 1995, p. 187.

11. V. N. Vragov, Boundary value problems for nonclassical equations of mathematical physics, Textbook for Universities, Novosibirsk: NGU (in Russian), 1983.

12. A. M. Nakhushev, Equations of Mathematical Biology, Textbook for Universities, Moscow: Vysshaya Shkola (in Russian), 1995.

13. S. G. Krein, Linear Differential Equations in a Banach Space, Moscow: Nauka (in Russian), 1966.

14. A. Ashyralyev and I. Muradov, On stability estimation of difference scheme of a first order of accuracy for hyperbolic-parabolic equations, Izv. Akad. Nauk Turkmenistan Ser. Fiz.-Tekhn. Khim. Geol. Nauk (in Russian), 1 (1996), 35-39.

15. A. Ashyralyev and I. Muradov, On difference schemes second order of accuracy for hyperbolic-parabolic equations, in: Modeling the Processes in Exploration of Gas Deposits and Applied Problems of Theoretical Gas Hydrodynamics, Ilim, Ashgabat (in Russian), (1998), 127-138.

16. A. Ashyralyev and M. B. Orazov, Theory of operators and the methods of solutions of boundary value problems for equations of mathematical physics, 8 years Turkmenistan's. Independence, Ilim, Ashgabat (in Russian), (1999), 222-228.

17. A. Ashyralyev and M. B. Orazov, The theory of operators and the stability of difference schemes for partial differential equations mixed types, Firat University, Fen ve Muh. Bilimleri Dergisi, 11(3) (1999), 249-252.

18. A. Ashyralyev and A. Yurtsever, On a nonlocal boundary-value problem for hyperbolicparabolic equations, in: Application of Mathematics in Engineering and Economics, 26, Heron Press and Technical University of Sofia, (2001), 79-85.

19. A. Ashyralyev and A. Yurtsever, On difference schemes for hyperbolic-parabolic equations, Functional Differential Equations, 7(3-4) (2000), 189-203.

20. A. Ashyralyev and A. Yurtsever, On a nonlocal boundary value problem for semilinear hyperbolic-parabolic equations, Nonlinear Analysis. Theory. Methods and Applications, 47 (2001), 3585-3592.

21. A. Ashyralyev and A. Yurtsever, A note on the second order of accuracy difference schemes for hyperbolic-parabolic equations in a Hilbert space, Proceedings of Dynamic Systems and Applications, 4 (2004), 556-563.

22. A. Ashyralyev and A. Yurtsever, A note on the second order of accuracy difference schemes for hyperbolic-parabolic equations, Applied Mathematics and Computation, textbf165 (2005), 517-537.

23. A. Ashyralyev and A. Yurtsever, On Difference Schemes for Hyperbolic-Parabolic Equations, Dynamical Systems and Applications Proceedings, (2004), 136-153. 
24. A. Ashyralyev and Y. Ozdemir, A note on the nonlocal boundary value problem for hyperbolic-parabolic equations, Functional Differential Equations, 12(1-2) (2005), 67-81.

25. H. O. Fattorini, Second Order Linear Differential Equations in Banach Spaces, Notas de Matematica, North-Holland, 1985.

26. S. Piskarev, and S. Y. Shaw, On certain operator families related to cosine operator function, Taiwanese Journal of Mathematics, 1(4) (1997), 3585-3592.

\author{
Allaberen Ashralyev ${ }^{1}$ and Yildirim Ozdemir ${ }^{1,2}$ \\ ${ }^{1}$ Department of Mathematics, \\ Fatih University, 34500 \\ Buyukcekmece, Istanbul, \\ Turkey \\ ${ }^{2}$ Department of Mathematics, \\ Gebze Institute of Technology, \\ 41400, Kocaeli, \\ Turkey \\ E-mail: yozdemir@fatih.edu.tr
}

